\title{
A humanized model of experimental autoimmune uveitis in HLA class II transgenic mice
}

\author{
Giuseppina Pennesi, ${ }^{1}$ Mary J. Mattapallil, ${ }^{1}$ Shu-Hui Sun,${ }^{1}$ Dody Avichezer, ${ }^{1}$ \\ Phyllis B. Silver, ${ }^{1}$ Zaruhi Karabekian, ${ }^{1}$ Chella S. David, ${ }^{2}$ Paul A. Hargrave, ${ }^{3}$ \\ J. Hugh McDowell, ${ }^{3}$ W. Clay Smith, ${ }^{3}$ Barbara Wiggert, ${ }^{4}$ Larry A. Donoso, ${ }^{5}$ \\ Chi-Chao Chan, ${ }^{1}$ and Rachel R. Caspi ${ }^{1}$ \\ ${ }^{1}$ Laboratory of Immunology, National Eye Institute, NIH, Bethesda, Maryland, USA \\ ${ }^{2}$ Department of Immunology, Mayo Clinic, Rochester, Minnesota, USA \\ ${ }^{3}$ Department of Ophthalmology, University of Florida, Gainesville, Florida, USA \\ ${ }^{4}$ Laboratory of Retinal Cell and Molecular Biology, National Eye Institute, NIH, Bethesda, Maryland, USA \\ ${ }^{5}$ Wills Eye Hospital, Philadelphia, Pennsylvania, USA
}

\begin{abstract}
Experimental autoimmune uveitis (EAU) is a disease of the neural retina induced by immunization with retinal antigens, such as interphotoreceptor retinoid-binding protein (IRBP) and arrestin (retinal soluble antigen, $\mathrm{S}-\mathrm{Ag}$ ). EAU serves as a model for human autoimmune uveitic diseases associated with major histocompatibility complex (HLA) genes, in which patients exhibit immunological responses to retinal antigens. Here we report the development of a humanized EAU model in HLA transgenic (TG) mice. HLA-DR3, -DR4, -DQ6, and -DQ8 TG mice were susceptible to IRBPinduced EAU. Importantly, HLA-DR3 TG mice developed severe EAU with S-Ag, to which wild-type mice are highly resistant. Lymphocyte proliferation was blocked by anti-HLA antibodies, confirming that antigen is functionally presented by the human MHC molecules. Disease could be transferred by immune cells with a Th1-like cytokine profile. Antigen-specific T cell repertoire, as manifested by responses to overlapping peptides derived from S-Ag or IRBP, differed from that of wild-type mice. Interestingly, DR3 TG mice, but not wild-type mice, recognized an immunodominant S-Ag epitope between residues 291 and 310 that overlaps with a region of S-Ag recognized by uveitis patients. Thus, EAU in HLA TG mice offers a new model of uveitis that should represent human disease more faithfully than currently existing models.
\end{abstract}

J. Clin. Invest. 111:1171-1180 (2003). doi:10.1172/JCI200315155.

\section{Introduction}

Experimental autoimmune uveitis (EAU) is a T cellmediated autoimmune disease model that targets the neural retina and related tissues. EAU is induced by immunization with preparations of purified retinal antigens or their fragments, of which the retinal soluble antigen ( $\mathrm{S}-\mathrm{Ag}$, also known as arrestin) and the interphotoreceptor retinoid-binding protein (IRBP) are the best known. This experimental model is used to represent a series of human inflammatory diseases

Received for publication January 28, 2002, and accepted in revised form February 11, 2003.

Address correspondence to: Rachel R. Caspi, Laboratory of Immunology, National Eye Institute, National Institutes of Health, 10 Center Drive, Bethesda, Maryland 20892, USA.

Phone: (301) 435-4555; Fax: (301) 480-6668;

E-mail: rcaspi@helix.nih.gov.

Giuseppina Pennesi's present address is: Laboratorio di Differenziamento Cellulare, Istituto Nazionale per la Ricerca sul Cancro, Genova, Italy.

Giuseppina Pennesi and Mary J. Mattapallil contributed equally to this work.

Conflict of interest: The authors have declared that no conflict of interest exists.

Nonstandard abbreviations used: experimental autoimmune uveitis (EAU); retinal soluble antigen (S-Ag); interphotoreceptor retinoid-binding protein (IRBP); transgenic (TG); bovine IRBP (bIRBP); bovine retinal soluble antigen (bS-Ag). collectively known as uveitis, which are characterized by a frequent presence of immune response to retinal antigens, particularly S-Ag. EAU can also be induced by adoptive transfer of $T$ cells from immunized recipients to naive, genetically compatible hosts (1). In most cases, the pathogenesis of the disease implicates a Th1type cytokine response (1).

Genetic studies have documented association between the presence or absence of certain HLA alleles and susceptibility to particular autoimmune disorders (2). Autoimmune uveitis is a spectrum of diseases that show differences in clinical manifestations and course, and differences in associations with specific HLA loci. The associated class I or class II alleles may also depend on the ethnic origin of the population studied. Among the class II-associated uveitic syndromes, the HLA-DRB $1 * 0405$ allele, encoding for a variant of the HLA-DR4 antigen, was found to be significantly increased in a Japanese population of Vogt-Koyanagi-Harada patients (3), and this was also confirmed in other populations $(4,5)$. Clinically similar to Vogt-Koyanagi-Harada syndrome, sympathetic ophthalmia is also associated with HLA-DR4 subtypes in Japanese, British, and Irish populations (6, 7). Intermediate uveitis not associated with multiple sclerosis was associated with the HLA-DR3 antigen, 
while panuveitis showed association with HLA-DR4 in an Italian population (8). Association with HLA-DQ alleles was also described $(3,7,9)$, but the strong linkage disequilibrium between some DR and DQ alleles makes it difficult to distinguish whether there was a prevalent role of one molecule or whether the DR and DQ genes acted in an epistatic manner. Genetic associations in uveitis have recently been reviewed in depth (10).

The antigen or antigens that might be involved in the etiology of human uveitis have not been elucidated. Although uveitis patients frequently display cellular and humoral responses to the retinal antigens that are uveitogenic in animals, it is not known whether these responses are causally involved in their disease or represent an epiphenomenon (11). This information is key for future development of antigen-specific therapies for these blinding autoimmune diseases. If indeed these responses are causally related, the immunodominant epitopes are expected to vary in different HLA types and will need to be defined in order for sophisticated therapies to be possible.

Similarly to uveitis in the human, EAU in animals is genetically controlled (10). There are clear species-specific differences in sensitivity to uveitogenic proteins. For example, IRBP is a much more potent uveitogen than S-Ag for mice, while the reverse is true in guinea pigs, and both proteins are strongly uveitogenic in the Lewis rat (12-15). Within each species, strain dependence of susceptibility is apparent that is at least in part due to MHC control. Susceptible H2 haplotypes are $\mathrm{H} 2^{\mathrm{b}}, \mathrm{H} 2^{\mathrm{d}}, \mathrm{H} 2^{\mathrm{k}}$, and $\mathrm{H} 2^{\mathrm{r}}$. MHC control of susceptibility to EAU in the $\mathrm{H} 2^{\mathrm{k}}$ haplotype was tentatively mapped to the IA subregion (a homologue of human HLA-DQ), with modifying effects from the IE locus (homologous to human HLA-DR) (15).

To better study the role of HLA molecules in the pathogenesis of uveitis, we undertook to develop a "humanized" model of EAU. To this end, we induced EAU in mice expressing transgenic (TG) HLA-DR3, -DR4, -DQ6, or -DQ8 molecules in the absence of the endogenous class II $(A \beta 0)(16)$. These mice positively select a repertoire of T cells expressing various $\mathrm{V} \beta \mathrm{T}$ cell receptors that can identify immunogenic peptide epitopes similar or identical to human subjects of the same HLA-DR or-DQ genotype $(16,17)$. We show that all the tested HLA TG mice developed EAU after immunization with IRBP. In addition, HLA-DR3 TG mice developed severe uveitis after immunization with $\mathrm{S}$-Ag, to which wild-type mice are highly resistant. Antibody blocking studies confirmed that the antigens were being recognized by the $T$ cells in the context of the human HLA molecules. Furthermore, recognition of peptides derived from S-Ag and peptides derived from IRBP by HLA TG mice differs from recognition by wild-type mice and has some similarity to that of uveitis patients. Finally, as in classical EAU, the disease can be transferred by immune cells having a Th1 phenotype, but not by immune serum.
This new humanized model of uveitis offers a more relevant approximation of human uveitis than hitherto available rodent models and will facilitate the characterization of uveitogenic epitopes presented by different HLA class II types.

\section{Methods}

Animals. HLA-DR4, -DQ6, and -DQ8 single TG mice were developed at the Mayo Clinic and have been described previously (18-20). HLA-DR3 mice originally developed by Günter Hammerling and associates (21) were bred onto the $A \beta 0$ background at the Mayo Clinic. The HLA-DR3 TG mice that carry the $H L A-D R A * 0103$ and $D R B 1 * 0301$ genes in an MHC class II-negative $\mathrm{H} 2-\mathrm{A} \beta 0$ background (A $\beta 0 . D R 3)$ express the human DR3 antigen as their only MHC class II molecule (22). HLA-DR4 TG mice had the HLA-DRB $1 * 0401$ gene in an A $\beta 0$ background, modified to express IE molecules by insertion of the gene for the IE $\alpha$ chain from the $\mathrm{H} 2^{\mathrm{k}}$ haplotype $\left(E \alpha^{k}\right)$ $E \beta^{b}$.A $\left.30 . D R 4\right)$ (18). Thus, they coexpressed murine H2-IE and human HLA-DR4 molecules. HLA-DQ6 TG mice carried the $D Q A 1 * 0103$ and $D Q B 1 * 0601$ transgenes in an $A \beta 0$ background (A $\beta 0 . D Q 6)$ (19). Similarly, HLA-DQ8 TG mice had the $D Q A 1 * 0301$ and $D Q B 1 * 0302$ human genes $(A \beta 0 . D Q 8)(20,23)$. Both HLA-DQ TG mice expressed the human DQ molecule as their only MHC class II antigen (Table 1). Controls were DR3- and DR4-negative littermates, $\mathrm{A} \beta 0$ mice, $A \beta 0$ mice expressing IE antigens $\left(E \alpha^{k} / E \beta^{b} . A \beta 0\right)$, and C57BL/10 or C57BL/6 mice. DR3-negative littermates did not express any MHC class II molecules, their genotype being equivalent to that of $A \beta 0$ mice. DR4negative littermates expressed the IE molecule, having a genotype comparable to $E \alpha^{k} / E \beta^{b} . A \beta 0$. These mice had the H2-IE molecule as their only MHC class II antigen. $\mathrm{H} 2-\mathrm{IA}^{\mathrm{b}}$ is the only MHC class II molecule expressed by the parental strain $\mathrm{C} 57 \mathrm{BL} / 10$, which has a defective gene encoding the $\mathrm{E} \beta$ chain and fails to express IE molecules (Table 1).

Animals were bred and maintained at the NIH or at the Mayo Clinic under specific pathogen-free conditions and were given water and chow ad libitum. The care and use of the animals was in compliance with institutional guidelines.

Genotyping. Mice were screened for presence of the human HLA molecule by PCR as described $(24,25)$. Expression of the human or murine MHC antigens was quantified by flow cytometric analysis of lymph node cells and splenocytes using FITC-conjugated anti-DR (clone L243), anti-DQ (clones Tü 39 and Tü 169), anti-IA (clone AF6-120.1), or anti-IE (clone 14-4-4S) antibodies (BD Pharmingen, Franklin Lakes, New Jersey, USA) as recommended by the manufacturer. Mice that did not demonstrate expression of HLA molecules by flow cytometry were excluded from analysis.

Antigens and reagents. Bovine IRBP (bIRBP) was purified from retinal extracts as described (26) by affinity 
chromatography on ConA followed by ion exchange chromatography on a Pharmacia MonoQ column. Bovine S-Ag (bS-Ag) was prepared from the ConA column flowthrough as follows. The extract was dialyzed against 10 volumes of $10 \mathrm{mM}$ HEPES, $15 \mathrm{mM} \mathrm{NaCl}, 1$ mM EDTA, $1 \mathrm{mM}$ benzamidine, $\mathrm{pH} 7.0$, with the buffer changed once. S-Ag was purified by the method of Buczylko and Palczewski (27) with modifications described by Puig et al. (28). The final elution from the heparin-agarose column was via a gradient from $10 \mathrm{mM}$ HEPES and $15 \mathrm{mM} \mathrm{NaCl}, \mathrm{pH} 7.0$, to $10 \mathrm{mM}$ HEPES and $400 \mathrm{mM} \mathrm{NaCl}, \mathrm{pH}$ 7.0. Purified bS-Ag was pooled based on an OD of $278 \mathrm{~nm}$. Preparation of the recombinant human S-Ag (hS-Ag) was described previously (29). Pertussis toxin and CFA were purchased from Sigma-Aldrich (St. Louis, Missouri, USA). Mycobacterium tuberculosis strain H37RA was purchased from Difco Laboratories (Detroit, Michigan, USA). Twenty-residue peptides overlapping by ten residues and spanning the first homologous repeat of human IRBP (hIRBP) (35 kDa) (30) and the entire hS-Ag (48 kDa; AnaSpec Inc., San Jose, California, USA) were synthesized by conventional solid-phase techniques as described $(30,31)$.

EAU induction and scoring. EAU induction and scoring was performed two ways: by active immunization and by adoptive transfer. For active immunization, mice were injected subcutaneously with $200 \mu \mathrm{g}$ of bIRBP, bS-Ag, or recombinant hS-Ag emulsified (1:1, vol/vol) with CFA (Sigma-Aldrich) that had been supplemented with $M$. tuberculosis strain H37RA (Difco Laboratories) to a final concentration of $2.5 \mathrm{mg} / \mathrm{ml}$. Concurrent with immunization, $0.2 \mu \mathrm{g}$ of pertussis toxin was injected intraperitoneally. Eyes from IRBPimmunized mice were collected 21-28 days after immunization, and eyes from S-Ag-immunized animals were collected after 28-35 days. Eyes were fixed for 1 hour in $4 \%$ phosphate-buffered glutaraldehyde and transferred into $10 \%$ phosphate-buffered formaldehyde. Fixed and dehydrated tissue was embedded in methacrylate. Sections (4-6 $\mu \mathrm{m})$ were cut through the pupillary-optic nerve plane and then stained with standard H\&E.

Quantitation of disease was performed in a masked fashion using criteria described previously (32). Briefly, eyes were assigned a score ranging from 0 to 4 depending on the extent of inflammation and tissue damage. The minimal criterion to score an eye as positive by histopathology was inflammatory cell infiltration of the ciliary body, choroid, vitreous, or retina (EAU grade 0.5). Progressively higher grades were assigned for the presence of discrete lesions in the tissue, such as vasculitis, granuloma formation, retinal folding and/or detachment and photoreceptor damage. The maximal grade of 4 reflects extensive retinal damage with complete destruction of the photoreceptor cell layer.

For induction of EAU by adoptive transfer, HLADQ8 and HLA-DQ6 mice were immunized with IRBP and HLA-DR3 mice were immunized with S-Ag using the uveitogenic protocol described above. On day 14 after immunization, immune serum was collected, titrated for antibody content by ELISA, and stored at $4^{\circ} \mathrm{C}$ until infusion into naive recipients. Lymph node and spleen cells were pooled within a group and were cultured for 3 days as described previously (33) with an optimal concentration of the immunizing antigen (30 $\mu \mathrm{g} / \mathrm{ml} \mathrm{IRBP}$ or $10 \mu \mathrm{g} / \mathrm{ml} \mathrm{S}-\mathrm{Ag}$ ) and $5 \mathrm{ng} / \mathrm{ml} \mathrm{recombi-}$ nant IL-12. Naive recipient mice of the appropriate genotype were infused intraperitoneally with $60 \times 10^{6}$ to $100 \times 10^{6}$ cultured cells. One milliliter of immune serum was injected intravenously as two doses of 0.5 ml administered concurrently with the cells and 48 hours later. Disease development was evaluated by fundoscopy and scored on a scale of $0-4$ as described previously (32). EAU was confirmed by histopathology in eyes harvested after 16-18 days.

Delayed-type hypersensitivity responses. Two days before the termination of an experiment, mice received $10 \mu \mathrm{g}$ of the appropriate antigen in a volume of $10 \mu \mathrm{l}$ intradermally into the pinna of one ear. The other ear was injected with PBS. Ear swelling was measured 48 hours later with a spring-loaded micrometer.

\section{Table 1}

MHC class II genotype and phenotype of mouse strains used in this study

\begin{tabular}{|c|c|c|c|c|}
\hline Strain & HLA transgene $\mathrm{A}^{\mathrm{A}}$ & MHC class II phenotype & MHC class II restriction element & Referenc \\
\hline \multicolumn{5}{|l|}{ Test strains } \\
\hline DR3 & $D R A^{*} 0103 ; D R B 1 * 0301$ & $\mathrm{~A} \beta 0 . \mathrm{DR}^{+}$ & HLA-DR3 & $(21)$ \\
\hline DR4 & $D R B 1 * 0401$ & $E \alpha^{k} / E \beta^{b} . A \beta 0 . D R 4^{+}$ & HLA-DR4/H2-IEk/b & (18) \\
\hline DQ6 & $D Q A 1 * 0103 ; D Q B 1 * 0601$ & $\mathrm{~A} \beta 0 . \mathrm{DQ}^{+}$ & HLA-DQ6 & (19) \\
\hline DQ8 & $D Q A 1 * 0301 ; D Q B 1 * 0302$ & $\mathrm{~A} \beta 0 . \mathrm{DQ} 8^{+}$ & HLA-DQ8 & $(20,23)$ \\
\hline \multicolumn{5}{|l|}{ Control strains } \\
\hline DR3- littermates & - & A $\beta 0 . D^{D} 3^{-}$ & - & \\
\hline DR4- littermates & - & $E \alpha^{k} / E \beta^{b} . A \beta 0 . D R 4^{-}$ & $\mathrm{H} 2-\mathrm{I} \mathrm{E}^{\mathrm{k} / \mathrm{b}}$ & \\
\hline$A \beta 0$ & - & $\mathrm{A} \beta 0$ & - & $(51)$ \\
\hline$E \alpha^{k} / E \beta^{b} . A \beta 0$ & - & $E \alpha^{k} / E \beta^{b} . A \beta 0$ & $\mathrm{H} 2-\mathrm{IE} / \mathrm{b}$ & $(52)$ \\
\hline $\mathrm{C} 57 \mathrm{BL} / 10$ or $\mathrm{C} 57 \mathrm{BL} / 6$ & - & $H 2-I A^{b}$ & $\mathrm{H} 2-\mathrm{IA}^{\mathrm{b}}$ & \\
\hline
\end{tabular}

AThe TG strains are on the $\mathrm{C} 57 \mathrm{BL} / 10$ background. 
a

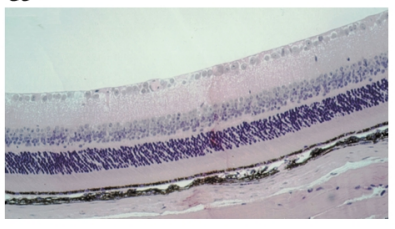

c

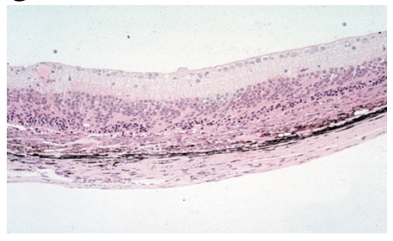

b

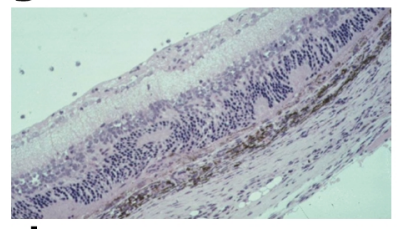

d

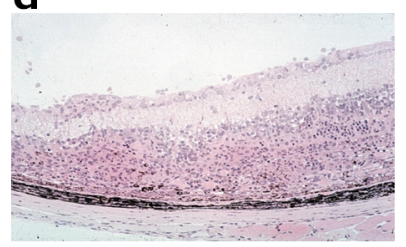

\section{Figure 1}

Histopathological features of EAU in HLA TG mice. (a) Normal eye. The retinal layers are ordered and well preserved. (b) Disease score 2 in C57BL/10 mice immunized with IRBP. The retinal architecture is disorganized, inflammatory cell infiltration is present, and the photoreceptor layer is damaged. (c) Disease score 3 in DR4 TG mice immunized with IRBP. Note extensive destruction of the photoreceptor cell layer. (d) Very severe disease (score 4) in DR3 TG mice immunized with S-Ag. Note complete destruction of the photoreceptor cell layers including photoreceptor cells (H\&E, ×200).

Lymphocyte proliferation assay and antibody blocking. Lymph nodes draining the immunization site (inguinal and iliac) were collected at the termination of each experiment and were pooled within each group. Triplicate $0.2-\mathrm{ml}$ cultures containing $5 \times 10^{5}$ cells/well were stimulated with $30 \mu \mathrm{g}$ of IRBP or $20 \mu \mathrm{g}$ of S-Ag in 96-well round-bottomed plates in RPMI 1640 (BioWhittaker Inc., Walkersville, Maryland, USA) supplemented as described and containing $1 \%$ mouse serum (32). For proliferation to bIRBP, 20 $\mathrm{mg} / \mathrm{ml} \alpha$-methyl-mannopyranoside (Sigma-Aldrich) was included to neutralize any possible traces of Con A that might leach from the column used in the initial stages of IRBP purification. This concentration of $\alpha$-methyl-mannopyranoside had no adverse effect on cell proliferation. Results are presented as stimulation indexes, calculated as average cpm of triplicate cultures with antigen, divided by the average cpm of triplicate cultures with medium, \pm SE.

The restriction element involved in antigen presentation was assessed by antibody blocking assays using purified antibodies to DR (clone L243), DQ (clone Tü 39 or Tü 169), IA (clone AF6-120.1), or IE (clone 14-44S) (BD Pharmingen). The antibodies were added at a concentration of $10 \mu \mathrm{g} / \mathrm{ml}$ to wells containing the cells in the presence or absence of the appropriate stimulating antigen. The cultures were incubated for 60 hours and were pulsed with ${ }^{3} \mathrm{H}$ thymidine $(1.0 \mu \mathrm{Ci} / 10 \mu \mathrm{l} /$ well $)$ for the last 18 hours.

$T$ cell epitope mapping. Twenty-residue peptides overlapping by 10 residues and representing the linear sequence of the first repeat of hIRBP or the entire sequence of the hS-Ag molecule were used (30) (AnaSpec Inc.). HLA-DQ8 TG, HLA-DR3 TG,

or wild-type C57BL/6 mice $\left(\mathrm{H} 2^{\mathrm{b}}\right)$ were immunized with a uveitogenic regimen of IRBP or S-Ag, respectively. Spleens were harvested on day 10 after immunization and were pooled from several mice within each group. Cells were tested in a standard proliferation assay, as described above, against a $10-\mu \mathrm{M}$ concentration of peptides derived from the immunizing antigen. Specific counts $(\Delta \mathrm{cpm})$ were calculated after subtraction of background cpm.

Determination of lymphokine content in culture supernatants and antibodies in immune serum by ELISA. Cytokines were determined in supernatants of lymph node cells collected on day 14 after immunization and cultured with an optimal concentration of the immunizing antigen as for the proliferation assays above. Supernatants were collected after 48 hours. The presence of IL-2, IL-4, IL-5, IL-6, IL-10, IL-12, IL-13, IFN- $\gamma$, and TNF- $\alpha$ in the supernatant of the stimulated cells was measured by multiplex ELISA using the Pierce SearchLight technology (Pierce Boston Technology, Woburn, Massachusetts, USA) (34) (http://www.searchlightonline.com). Serum antibodies were assayed on IRBP- or S-Ag-coated plates $(5 \mu \mathrm{g} / 100 \mu \mathrm{l} /$ well $)$ using HRP-labeled goat anti-mouse IgG (Zymed Laboratories Inc., San Francisco, California, USA) as developing antibody and 3,3'5,5' -tetramethylbenzidine substrate (100 $\mu \mathrm{l} /$ well; Endogen Inc., Woburn, Massachusetts, USA).

Statistical analysis. Statistical significance of differences in disease scores were calculated using Snedecor and Cochran's test (35) for linear trend in proportions, with each mouse (average of both eyes) as one statistical event. This is a nonparametric test that generates its $P$ values by frequency analysis of the number of individuals at each possible score, thus taking into account both severity and incidence of disease. Delayed hypersensitivity and lymphocyte

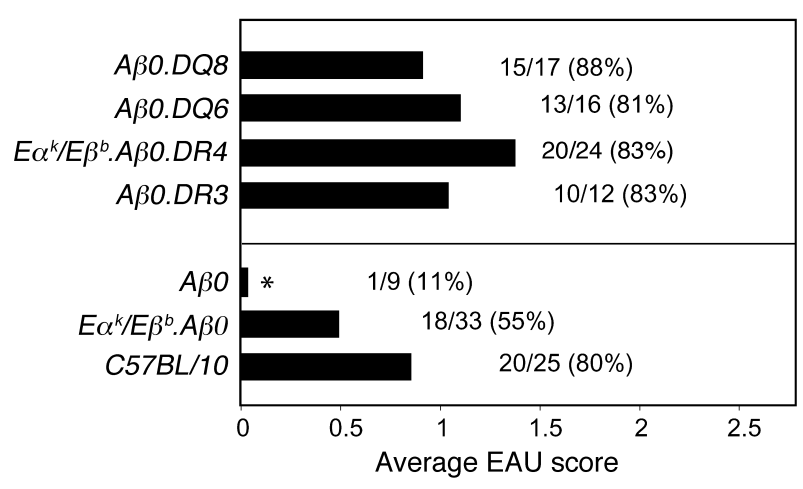

\section{Figure 2}

Susceptibility of HLA TG mice to IRBP-induced EAU. Bars are average of disease scores. Disease incidence (positive among total mice) is next to each bar. The HLA TG strains are in the top panel and the control strains are in the bottom panel. If only one eye showed disease the animal was scored as positive and its score was recorded as the average of both eyes. Shown are combined results of five experiments. Significant difference $(P \leq 0.05)$ from $C 57 \mathrm{BL}$ control is indicated by an asterisk. 


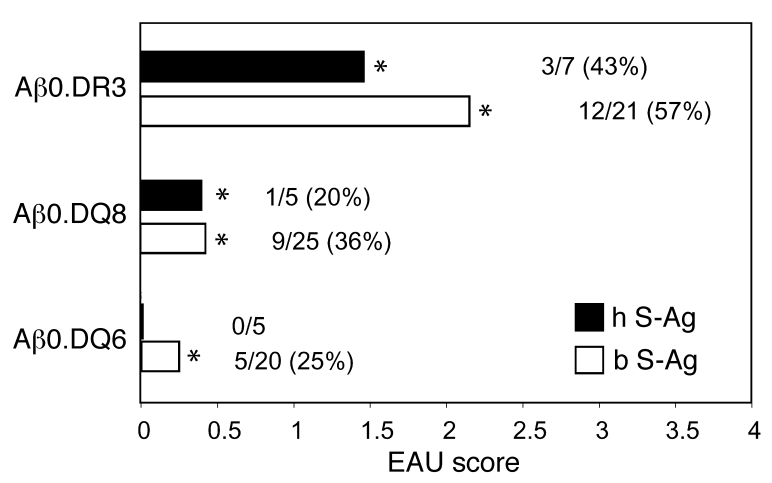

Figure 3

Susceptibility of HLA TG mice to S-Ag-induced EAU. Shown are combined results of five experiments using bS-Ag and one experiment using recombinant $\mathrm{hS}-\mathrm{Ag}$. Other TG strains were negative. Control $A \beta 0$ mice immunized with bS-Ag $(n=6)$ and wild-type C57BL/10 mice immunized with either hS-Ag $(n=4)$ or bS-Ag $(n=19)$ failed to develop disease. Significant differences $(P \leq 0.05)$ from $C 57 \mathrm{BL}$ and $A \beta 0$ controls are indicated by asterisks.

proliferation data were analyzed using an independent $t$ test. Probability values of $P \leq 0.05$ were considered to be significant.

\section{Results}

HLA TG mice are susceptible to IRBP-induced uveitis. HLA TG mice were immunized with a uveitogenic protocol of IRBP and eyes were harvested for histopathology 21-28 days after immunization. Typical EAU was induced in all HLA TG strains. Histopathology (Figure $1, \mathrm{~b}$ and c) was grossly similar to that observed in wild-type mice on the C57BL/10 background, showing mononuclear and polymorphonuclear cell infiltration, vitritis, choroiditis, and varying degrees of photoreceptor cell damage. Disease severity in individual mice varied from 0.5 to 3 , and incidence was about $80 \%$ (Figure 2). These results were not different from incidence and severity scores observed in the control strains. $A \beta 0$ mice, which do not express any MHC class II molecules but do express murine class I molecules, had little to no disease, with only one mouse of 11 showing trace infiltration in one eye, most likely representing a mild spontaneous inflammation unrelated to the experimental manipulations. We do not favor the possibility that this represents presentation of IRBP through the class I pathway because these mice had negligible IRBPspecific delayed-type hypersensitivity and proliferative responses (data not shown), suggesting that in the absence of class II, IRBP immunization fails to elicit a significant response.

Since no apparent differences in disease parameters were observed in HLA TG mice expressing or not expressing the murine IE

Figure 4 molecules, their role when a human class II molecule is present appears to be subordinate. Nevertheless, when a human class II is absent, such as in the $E \alpha^{k} / E \beta^{b} . A \beta 0$ mice, the presence of murine IE alone is sufficient to permit EAU development.

DR3 TG mice are susceptible to $S$-Ag-induced EAU. Uveitis patients frequently exhibit strong lymphocyte responses to $\mathrm{S}-\mathrm{Ag}$ but only sporadic responses to IRBP $(12,36)$. Although S-Ag is uveitogenic in other animal species, numerous strains of mice tested thus far proved to be highly refractory to disease induction with S-Ag $(14,15)$. Interestingly, HLA-DR3 TG mice immunized with bS-Ag developed very severe disease, with many individual mice reaching disease scores of 4, corresponding to complete destruction of the photoreceptor cell layer and extensive damage to the other retinal layers (Figure 1d and Figure 3). HLA-DQ6 and -DQ8 mice developed some disease as well, albeit with much lower scores and lower incidence (Figure 3 ). None of the other TG strains or any of the six control $A \beta 0$ mice developed disease after immunization with S-Ag (data not shown). Wild-type mice were also refractory to disease, confirming previously published data (14) (not shown). Onset of S-Ag-induced disease in the DR3 TG mice, which characteristically developed high disease scores, appeared to be delayed by several days to a week in comparison to the IRBP-induced disease. This is similar to the earlier onset of IRBP-induced EAU than of S-Ag EAU as observed in rats, and may reflect a difference in accessibility of the target antigen (S-Ag is intracellular in the photoreceptor cells, whereas IRBP is secreted into the interphotoreceptor matrix) (37).

There is about $80 \%$ homology in the amino acid sequence between human and bS-Ag. To test whether this translated to differences in pathogenicity, we immunized HLA TG mice with recombinant hS-Ag.

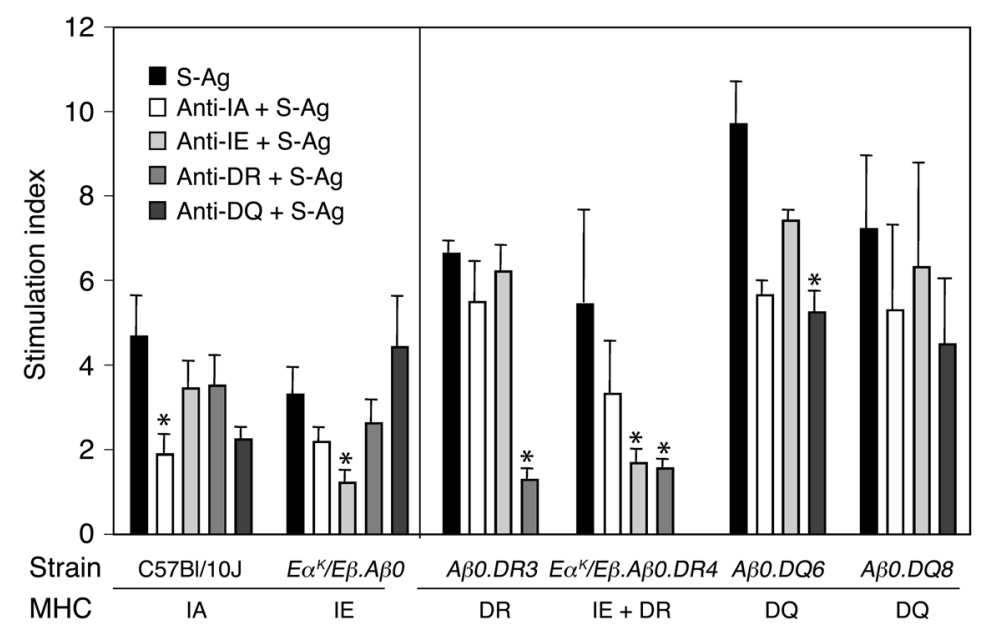

Proliferative response of lymph node cells to bS-Ag. Average of stimulation indices observed in several experiments is shown. Error bars indicate SE. Significant difference from control $(P \leq 0.05)$ is indicated by an asterisk. Background counts varied between 1,500 and 3,000 cpm depending on the group. 
a

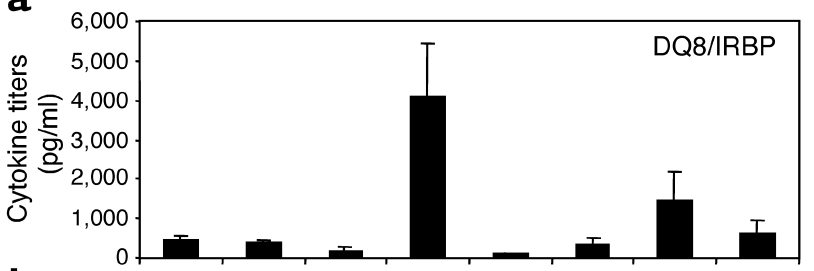

b

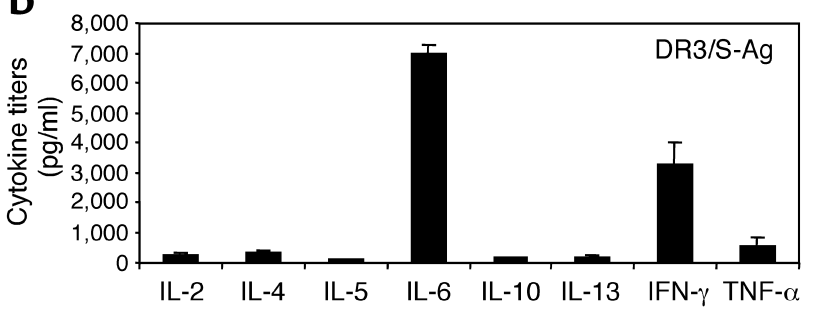

\section{Figure 5}

Cytokine responses. Cytokine production by lymph node cells from HLA-DQ8 TG mice stimulated with bIRBP (a), and lymph node cells from HLA-DR3 TG mice stimulated with bS-Ag (b), measured by multiplexed ELISA. Shown are titers in $\mathrm{pg} / \mathrm{ml}$ averaged from two experiments.

DR3 and DQ8 TG mice developed EAU after immunization with the recombinant hS-Ag with scores similar to the EAU developed in response to bS-Ag (Figure 3). This finding is of particular importance in view of the strong responses of human patients to $\mathrm{S}-\mathrm{Ag}$, that had presumably developed as responses to the autologous human molecule. HLA-DR4 TG mice and all the control strains, including the wild type, were resistant (data not shown).

The retinal antigen is presented and recognized on buman MHC molecules. Because DR (though not DQ) strains retain the mouse $\mathrm{E} \beta$, and in the case of DR4 also the $\mathrm{E} \alpha$ molecule, it was necessary to verify that antigen can be presented productively on the human class II molecules. This was done by lymphocyte proliferation blocking studies using appropriate monoclonal antibodies. Draining lymph node cells from mice immunized with IRBP or bS-Ag were collected and were stimulated in culture with the corresponding antigen. Proliferative responses were observed in all strains except $A \beta 0$, which is devoid of any class II molecules. In HLA-DR3, -DQ6, and -DQ8 TG mice, proliferation in response to $\mathrm{S}-\mathrm{Ag}$ was blocked by specific monoclonal antibodies to human, but not to mouse, MHC class II molecules (Figure 4). In E $\alpha^{k} / E \beta^{b}$.A $\beta 0 . D R 4 T G$ mice, which coexpress DR and IE molecules, both anti-DR and anti-IE antibodies abrogated proliferation, indicating that both class II molecules were functionally involved in antigen presentation. A similar pattern was observed with HLA TG lymph node cells of mice immunized with IRBP (data not shown). A cytotoxic effect of antibodies was excluded because the anti-HLA antibodies did not interfere with proliferation of wildtype lymphocytes to antigen and because background counts in the presence of antibodies without antigen did not differ from background in presence of medium alone (data not shown).

It should be pointed out that strong proliferative responses were seen in all genotypes except $A \beta 0$, irrespective of whether or not they developed disease. Similarly, all strains except A $\beta 0$ mounted delayedtype hypersensitivity responses against $\mathrm{S}-\mathrm{Ag}$ and IRBP (data not shown). This indicates that not only pathogenic but also nonpathogenic epitopes of the retinal antigens in question were functionally presented by human class II molecules.

Pathogenesis of EAU in HLA TG mice: a cell-mediated response with a Th1-predominant cytokine profile and a modifying role for antibodies. Studies of EAU in wild-type mice indicated a central role for a Th1-type response in pathogenesis of disease (1). To evaluate the responses of HLA TG mice to uveitogenic immunization, we measured the cytokines in supernatants of lymph node and spleen cells from immunized mice that were stimulated in vitro with the immunizing antigen. We selected as representative strains the DQ8 TG mice
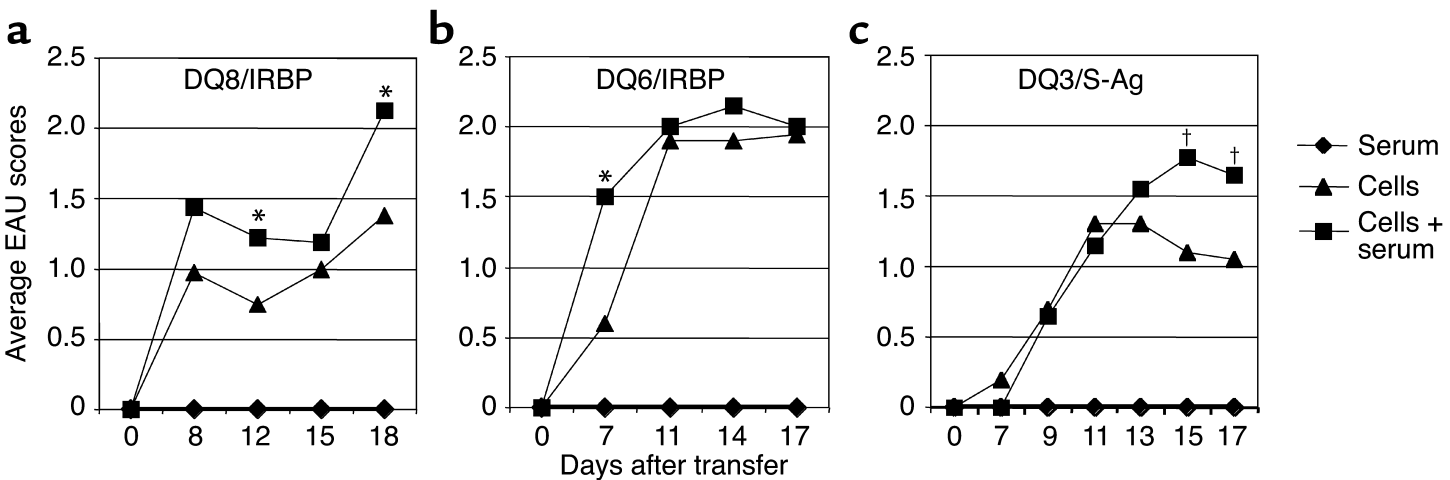

Figure 6

Adoptive transfer of cells and/or serum. Recipient mice were infused with serum, cells, or cells and serum from immunized syngeneic donors. EAU development was followed by fundus examination. ( $\mathbf{a}$ and $\mathbf{b}$ ) Disease scores of HLA-DQ8 and HLA-DQ6 recipients whose donors were immunized with bIRBP. (c) Disease scores of HLA-DR3 recipients whose donors were immunized with bS-Ag. Shown is one of two representative experiments with five mice per group. *Statistically significant difference in scores from cells alone $(P \leq 0.05)$. ${ }^{\dagger}$ Trend $(P \leq 0.1)$. 

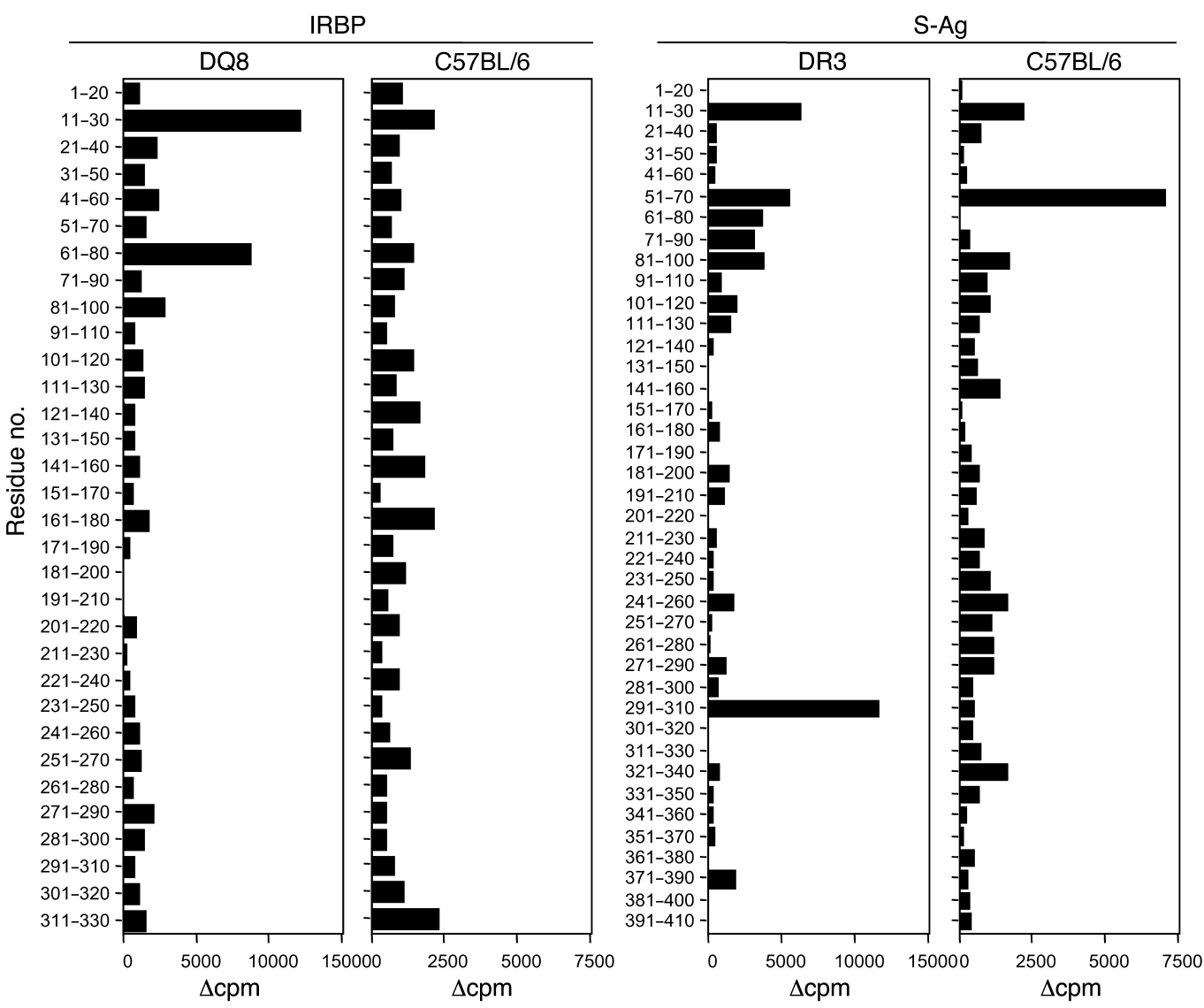

Figure 7

Mapping of T cell epitopes. Epitope recognition by DQ8 mice immunized with bIRBP and DR3 TG mice immunized with bS-Ag was compared with that of $\mathrm{C} 57 \mathrm{BL} / 6\left(\mathrm{I} \mathrm{A}^{\mathrm{b}}\right)$ wild-type controls. Antigen-specific responses were recalled with overlapping peptides representing the first repeat of human IRBP or the whole hS-Ag. Shown are specific counts as averaged from two to three repeat experiments for each strain of mice. Background counts varied from 450 to $3,000 \mathrm{cpm}$ depending on the group.

immunized with bIRBP and the DR3 TG mice immunized with bS-Ag, which were high responders for the respective antigens. Both strains produced very high levels of IL-6, which was also typical of wild-type C57BL/6 mice (not shown). Of the cytokines typifying a Th1 or a Th2 response, IFN- $\gamma$ and TNF- $\alpha$ were predominant over IL-4, IL-5, and IL-13. DR3 mice produced relatively more IFN- $\gamma$ than did DQ8 mice (Figure 5 , a and $b$ ). Thus, EAU induced in HLA TG mice appears to be associated with a Th1-predominant cytokine response profile, analogous to the "classical" model of EAU in wild-type mice.

In wild-type mice, EAU can be adoptively transferred by immune cells but not by immune serum. To test whether the pathogenesis of EAU in HLA TG mice was cell- or antibody-mediated, we adoptively transferred cells, serum, or cells and serum together from immunized to naive syngeneic mice which were then followed by fundus examination. Activated lymphocytes from immunized mice cultured with antigen in the presence of IL-12 (33) were able to transfer EAU to naive syngeneic recipients (Figure 6). None of the mice that received high-titer immune serum alone (antibody titers between $1 \times 10^{-6}$ and $1 \times 10^{-7}$, see
Methods) developed disease. Interestingly, when cells and serum were combined, the serum was able to slightly but consistently raise disease scores compared with cells alone, suggesting that antibodies can modify the course of disease.

$T$ cell epitope recognition of IRBP and S-Ag epitopes in HLA TG mice. The results described above indicated that $\mathrm{T}$ cells responding to retinal antigen presented on HLA molecules were involved in the disease process in HLA TG mice. Because the class II-restricted $\mathrm{T}$ cell repertoire is selected in these mice by human class II, we decided to examine epitope recognition of HLA TG mice. We chose as representative strains those with high disease susceptibility for each antigen: DQ8 for IRBP and DR3 for S-Ag. Mice were immunized with native bovine antigen, and spleen cell responses were recalled in vitro with a panel of overlapping peptides representing the entire first repeat of hIRBP or the entire sequence of hS-Ag. The responses were compared with those of $\mathrm{H} 2^{\mathrm{b}}$ haplotype wild-type mice (C57BL/6). Our decision to immunize with bovine proteins and recall with human peptides (although human recombinant proteins are available) was based on the reasoning that 
in a human-human or a bovine-bovine combination, the strongest responses would represent recognition of nonconserved epitopes foreign to the mouse. In the bovine-human combination, the responses would be more likely to represent recognition of conserved epitopes shared with the autologous antigen.

The results showed distinct differences between epitope recognition of HLA TG and wild-type mice, suggesting that a different $T$ cell repertoire had been selected (Figure 7). IRBP peptides 11-30 and 61-80 were immunodominant in DQ8TG mice but not in wild-type mice. S-Ag peptide 291-310 (NRERRGIALDGKIKHEDTNL) appeared to be immunodominant in DR3TG mice. This peptide was weakly recognized by wild-type mice, which instead responded strongly to peptide 51-70. It is of interest to note that peptide 291-310 of S-Ag partly overlaps with the previously characterized S-Ag peptides M 303-320 and $\mathrm{N}$ 281-302, which elicit responses in lymphocytes of patients affected by different uveitic diseases $(11,31)$.

\section{Discussion}

The present study describes a new, "humanized" model of EAU developed in HLA TG mice in which disease-relevant epitopes appear to be largely restricted by the human class II molecules. Evidence that this is indeed the case is provided by (a) lack of class II molecules other than the human one in the HLA-DQ6, HLA-DQ8, and HLA-DR3 mice; (b) lack of disease in the control $A \beta 0$ mice lacking the human class II; (c) the ability of the appropriate anti-HLA antibodies to block lymphocyte proliferation of HLA TG mice in response to the immunizing retinal antigen; and (d) distinct differences in epitope recognition between HLA TG and wild-type mice, suggesting differences in the selected Ag-specific repertoire.

Ours is not the first attempt at establishing a humanized model of uveitis. Uveitis is a heterogeneous group of diseases affecting different parts of the eye and showing association with various HLA class I or class II molecules (38). HLA-B27 is strongly associated with anterior uveitis that accompanies ankylosing spondylitis. HLA-A29 confers an increased risk of birdshot chorioretinopathy that is in some cases up to 224 times higher than the general population (39). Sympathetic ophthalmia is associated with HLA-DR4, and intermediate uveitis (unrelated to multiple sclerosis) with HLA-DR3 $(3-8,39$, 40). The relevant human HLA molecule does not necessarily precipitate the appearance of symptoms in the animal carrying it as a transgene. Although HLA-B27 TG rats and mice do develop spontaneous spondyloarthropathies, they do not develop either spontaneous or bacterially induced anterior uveitis (41-43). In contrast, the recently described HLA-A29 TG mice develop spontaneous uveitis with striking histological similarity to the HLA-A29-associated disease in humans. This supports the conclusion that the HLA-A29 molecule itself participates in the pathogenesis of birdshot chorioretinopathy (41). The EAU model in HLA class II TG mice described here provides evidence in favor of an involvement of class II-restricted responses in human uveitis. No less importantly, this new model suggests that the same retinal antigens that are uveitogenic in animals may also be causally involved in human uveitic diseases and validates antigen-specific immunotherapies based on these antigens, such as the recent oral tolerance trial in which uveitis patients were fed S-Ag and in fact appeared to receive a clinical benefit (44).

Arguably the most interesting observation in the present study is the susceptibility of HLA-DR3 TG mice to disease induced with S-Ag. Of particular note is the finding that the $\mathrm{T}$ cell epitope recognition in these mice takes on a similarity to that of uveitis patients. DR3 TG mice but not wild-type mice responded to S-Ag peptide 291-310. This sequence partly overlaps with peptide $\mathrm{M}$, a promiscuous $\mathrm{S}-\mathrm{Ag}$ epitope spanning residues 303-320 that is uveitogenic in rats, guinea pigs, and primates, and is recognized by lymphocytes from uveitis patients but not by healthy controls $(11,12,31,45)$. Although human patients appear to exhibit much more frequent cellular responses to S-Ag than to IRBP, wild-type mice develop EAU with IRBP but are highly resistant to EAU induced with S-Ag. It has been proposed that the reason for this is central tolerance to S-Ag, owing to its abundance in the murine thymus (46), such that $\mathrm{T}$ cells capable of recognizing S-Ag are efficiently deleted from the repertoire. The dramatic change in susceptibility of HLA-DR3 TG mice to S-Ag, apparently stemming solely from substitution of the restricting class II molecule from mouse to human, suggests that other mechanisms may be at play. Thus, in addition to and independently of its potential to bring better understanding of the involvement of S-Ag in human uveitic disease, the uveitis model in HLA-DR3 mice also offers the opportunity to approach basic questions in the development of selftolerance to retinal antigens. These questions will be the subject of a separate study.

The $E \alpha^{k} / E \beta^{b} . A \beta 0$ mice (which served here as controls for the $E \alpha^{\mathrm{k}} / \mathrm{E} \beta^{\mathrm{b}}$.A $\beta 0 . D R 4$ TG mice) provide some new information about the role of IE molecules in the EAU model. It is apparent that IA molecules are sufficient to permit induction of EAU in the $\mathrm{H} 2^{\mathrm{b}}$ haplotype, as mice on the C57BL background, which do not express IE molecules, develop disease. Our previous data in intra- $\mathrm{H} 2$ congenics tentatively mapped control of EAU to IA ${ }^{\mathrm{k}}$, with only modifying influence from $I^{k}(15)$. The present data show for the first time that the IE molecule by itself, without the presence of IA, is sufficient to confer the ability to develop EAU.

The interaction between the $\mathrm{T}$ cell coreceptor CD4 and MHC class II molecules plays a crucial role in intrathymic selection as well as peripheral activation of $\mathrm{CD}^{+} \mathrm{T}$ cells. In our humanized EAU model, 
murine CD4 molecules bind to the $\beta 2$ domain of human MHC class II. It has been argued that speciesspecific amino acid differences might alter the interaction between mouse CD4 and human class II, resulting in reduced or modified responses $(18,47)$. Since we do not have human CD4 TG mice at our disposal, we attempted to partly address the effect of a homologous versus heterologous CD4-class II interaction by using DR4 chimeric mice. These mice express a hybrid DR4 molecule formed from the invariant portion of the murine IE molecule that contains the CD4 binding site, fused with the variable region that contains the antigen-binding pocket of the human HLA-DR4 molecule. Unlike $E \alpha^{k} / E \beta^{b} . A \beta 0 . D R 4$ mice, these DR4 chimeric mice have no murine class II molecules and thus have only a homologous CD4-class II interaction. DR4 chimeric mice developed IRBP-induced EAU with an incidence and average score similar to that of nonchimeric DR4 TG mice and were resistant to S-Ag (data not shown). Thus, presence of a heterologous CD4-class II interaction in our model appears not to cause an altered pattern of susceptibility to disease.

The pathogenesis of EAU in the HLA TG mice appears to be similar to that of EAU in wild-type mice and reinforces the notion that cell-mediated responses may also be causally involved in human uveitis. In wild-type mice, uveitogenicity is associated with the Th1 response (1). Both IRBP-induced EAU in DQ8 TG mice and S-Ag-induced EAU in DR3 TG mice were associated with an IFN- $\gamma$-dominant response to their respective uveitogen. Interestingly, IL-2- and IFN- $\gamma-$ producing $\mathrm{CD}^{+}$and $\mathrm{CD} 8^{+}$cells are detected in peripheral blood from patients with Behçet disease $(48,49)$. Disease could be transferred with Th1-polarized cells from primed individuals into naive recipients. Immune serum alone did not transfer disease, but in conjunction with the cells, injection of serum was able to slightly, but consistently, raise disease scores. This suggests that although the antibodies by themselves are unable to enter the eye through an intact bloodretinal barrier, once the blood-retinal barrier is breached by activated $\mathrm{T}$ cells, antibodies serve to modify the course of disease.

In summary, this is the first report of a humanized class II-restricted model of uveitis that includes a new mouse model for S-Ag-induced uveitis. This model validates the class II involvement in human uveitis and supports an etiological role for retinal antigens, which are uveitogenic in animals, in human disease. Identification of $\mathrm{T}$ cell determinants restricted to MHC molecules known to predispose to uveitis will permit better understanding of disease mechanisms and promises to facilitate development of antigen-specific therapies tailored to particular HLA haplotypes. Importantly, once autoantigenic epitopes have been identified, the disease model generated in these mice will permit manipulations to mechanistically elucidate the development of autoimmunity that are not possible in human patients (50).

\section{Acknowledgments}

We thank Julie Hanson and her staff at the Immunogenetic Mouse Colony, Mayo Clinic, for providing some of the HLA-TG mice used in this study. We thank Rajeev Agarwal and Angelia Viley for support in genotyping the HLA TG mice bred at NIH. We are grateful to Rafael Grajewski for assistance in fundoscopic evaluations and useful discussions, and to Shao-Bo Su for flawless intravenous injections.

1. Caspi, R.R. 2002. Th1 and Th2 responses in pathogenesis and regulation of experimental autoimmune uveoretinitis. Int. Rev. Immunol. 21:197-208.

2. Klein, J., and Sato, A. 2000. The HLA system - second of two parts. N. Engl. J. Med. 343:782-786.

3. Shindo, Y., Inoko, H., Yamamoto, T., Nakamura, S., and Ohno, S. 1994. HLA-DRB1 typing of Vogt-Koyanagi-Harada's disease by PCR-RFLP and the strong association with DRB $1 * 0405$ and DRB $1 * 0410$. Br.J. Ophthalmol. 78:223-226.

4. Alaez, C., et al. 1999. Strong association of HLA class II sequences in Mexicans with Vogt-Koyanagi-Harada's disease. Hum. Immunol. 60:875-882.

5. Kim, M.H., et al. 2000. Association of HLA with Vogt-Koyanagi-Harada syndrome in Koreans. Am. J. Ophthalmol. 129:173-177.

6. Shindo, Y., et al. 1997. Immunogenetic study of sympathetic ophthalmia. Tissue Antigens. 49:111-115.

7. Kilmartin, D.J., et al. 2001. Immunogenetics and clinical phenotype of sympathetic ophthalmia in British and Irish patients. Br.J. Ophthalmol. 85:281-286.

8. Cuccia Belvedere, M., et al. 1986. Genetic heterogeneity in uveitis. Dis. Markers. 4:243-246.

9. Melin-Aldana, H., et al. 1992. Human leukocyte antigen-DRB1*1104 in the chronic iridocyclitis of pauciarticular juvenile rheumatoid arthritis. J. Pediatr. 121:56-60.

10. Pennesi, G., and Caspi, R.R. 2002. Genetic control of susceptibility in clinical and experimental uveitis. Int. Rev. Immunol. 21:67-88.

11. Adamus, G., and Chan, C.C. 2002. Experimental autoimmune uveitides: multiple antigens, diverse diseases. Int. Rev. Immunol. 21:209-230.

12. Gery, I., Mochizuki, M., and Nussenblatt, R.B. 1986. Retinal specific antigens and immunopathogenic processes they provoke. In Progress in retinal research. Volume 5. N. Osborne and G.J. Chader, editors. Pergamon Press. Oxford, United Kingdom. 5:75-109.

13. Faure, J.P. 1980. Autoimmunity and the retina. Curr. Top. Eye Res. 2:215-301.

14. Caspi, R.R., et al. 1988. A new model of autoimmune disease. Experimental autoimmune uveoretinitis induced in mice with two different retinal antigens. J. Immunol. 140:1490-1495.

15. Caspi, R.R., Grubbs, B.G., Chan, C.C., Chader, G.J., and Wiggert, B. 1992. Genetic control of susceptibility to experimental autoimmune uveoretinitis in the mouse model. Concomitant regulation by MHC and nonMHC genes. J. Immunol. 148:22384-22389.

16. Taneja, V., and David, C.S. 1998. HLA transgenic mice as humanized models of disease and immunity. J. Clin. Invest. 101:921-926.

17. Yang, H., et al. 2002. Mapping myasthenia gravis-associated T cell epitopes on human acetylcholine receptors in HLA transgenic mice. J. Clin. Invest. 109:1111-1120. doi:10.1172/JCI200214255.

18. Pan, S., Trejo, T., Hansen, J., Smart, M., and David, C.S. 1998. HLA-DR4 (DRB1*0401) transgenic mice expressing an altered CD4-binding site: specificity and magnitude of DR4-restricted T cell response. J. Immunol. 161:925-929.

19. Kong, Y.M., et al. 1997. Role of mouse and human class II transgenes in susceptibility to and protection against mouse autoimmune thyroiditis. Immunogenetics. 46:312-317.

20. Neeno, T., et al. 1996. HLA-DQ8 transgenic mice lacking endogenous class II molecules respond to house dust allergens: identification of antigenic epitopes. J. Immunol. 156:3191-3195.

21. Strauss, G., Vignali, D.A., Schonrich, G., and Hammerling, G.J. 1994. Negative and positive selection by HLA-DR3(DRw17) molecules in transgenic mice. Immunogenetics. 40:104-108.

22. Kong, Y.C., et al. 1996. HLA-DRB1 polymorphism determines susceptibility to autoimmune thyroiditis in transgenic mice: definitive association with HLA-DRB1*0301 (DR3) gene. J. Exp. Med. 184:1167-1172.

23. Nabozny, G.H., et al. 1996. HLA-DQ8 transgenic mice are highly susceptible to collagen-induced arthritis: a novel model for human polyarthritis. J. Exp. Med. 183:27-37.

24. Olerup, O., and Zetterquist, H. 1992. HLA-DR typing by PCR amplification with sequence-specific primers (PCR-SSP) in 2 hours: an alternative to serological DR typing in clinical practice including donor-recipient matching in cadaveric transplantation. Tissue Antigens. 39:225-235. 
25. Olerup, O., Aldener, A., and Fogdell, A. 1993. HLA-DQB1 and -DQA1 typing by PCR amplification with sequence-specific primers (PCR-SSP) in 2 hours. Tissue Antigens. 41:119-134.

26. Pepperberg, D.R., Okajima, T.L., Ripps, H., Chader, G.J., and Wiggert, B. 1991. Functional properties of interphotoreceptor retinoid-binding protein. Photochem. Photobiol. 54:1057-1060.

27. Buczylko, J., and Palczewski, K. 1993. Purification of arrestin from bovine retinas. In Photoreceptor cells. P.A. Hargrave, editor. Academic Press Inc. San Diego, California, USA. 226-236.

28. Puig, J., et al. 1995. Synthetic phosphopeptide from rhodopsin sequence induces retinal arrestin binding to photoactivated unphosphorylated rhodopsin. FEBS Lett. 362:185-188.

29. Smith, W.C. 1996. A splice variant of arrestin from human retina. Exp. Eye Res. 62:585-592.

30. Donoso, L.A., et al. 1989. Human interstitial retinoid binding protein. A potent uveitopathogenic agent for the induction of experimental autoimmune uveitis. J. Immunol. 143:79-83.

31. de Smet, M.D., et al. 1990. Cellular immune responses of patients with uveitis to retinal antigens and their fragments. Am. J. Ophthalmol. 110:135-142.

32. Caspi, R.R. 1997. Experimental autoimmune uveoretinitis in the rat and mouse. In Current protocols in immunology. Volume 3. J.E. Coligan, A.M Kruisbeek, D.H. Margulies, E.M. Shevach, and W. Strober, editors. John Wiley and Sons Inc. Hoboken, New Jersey, USA. 15.6.1.

33. Tarrant, T.K., Silver, P.B., Chan, C.C., Wiggert, B., and Caspi, R.R. 1998 Endogenous IL-12 is required for induction and expression of experimental autoimmune uveitis. J. Immunol. 161:122-127.

34. Moody, M.D., Van Arsdell, S.W., Murphy, K.P., Orencole, S.F., and Burns, C. 2001. Array-based ELISAs for high-throughput analysis of human cytokines. Biotechniques. 31:186-190, 192-194.

35. Snedecor, G.W., and Cochran, W.G. 1967. Statistical methods. Iowa State University Press. Ames, Iowa, USA. 248 pp.

36. Hirose, S., et al. 1988. Lymphocyte responses to retinal-specific antigens in uveitis patients and healthy subjects. Curr. Eye Res. 7:393-402.

37. Fox, G.M., et al. 1987. Experimental autoimmune uveoretinitis (EAU) induced by retinal interphotoreceptor retinoid-binding protein (IRBP): differences between EAU induced by IRBP and by S-antigen. Clin. Immunol. Immunopathol. 43:256-264.

38. Nussenblatt, R.B., Whitcup, S.M., and Palestine, A.G. 1996. Uveitis: fundamentals and clinical practice. 2nd edition. Mosby-Year Book Inc. St. Louis, Missouri, USA. 22-26.

39. Baarsma, G.S., Priem, H.A., and Kijlstra, A. 1990. Association of birdshot retinochoroidopathy and HLA-A29 antigen. Curr. Eye Res. 9:63-68.

40. Feltkamp, T.E. 1990. Ophthalmological significance of HLA associated uveitis. Eye. 4:839-844.

41. Szpak, Y., et al. 2001. Spontaneous retinopathy in HLA-A29 transgenic mice. Proc. Natl. Acad. Sci. U. S. A. 98:2572-2576.

42. Baggia, S., et al. 1997. A novel model of bacterially-induced acute anterior uveitis in rats and the lack of effect from HLA-B27 expression. J. Investig. Med. 45:295-301.

43. Khare, S.D., Bull, M.J., Hanson, J., Luthra, H.S., and David, C.S. 1998. Spontaneous inflammatory disease in HLA-B27 transgenic mice is independent of MHC class II molecules: a direct role for B27 heavy chains and not B27-derived peptides. J. Immunol. 160:101-106.

44. Nussenblatt, R.B., et al. 1997. Treatment of uveitis by oral administration of retinal antigens: results of a phase I/II randomized masked trial. Am. J. Ophthalmol. 123:583-592.

45. Hirose, S., et al. 1989. An 18-mer peptide derived from the retinal S antigen induces uveitis and pinealitis in primates. Clin. Exp. Immunol. 77:106-111.

46. Egwuagu, C.E., Charukamnoetkanok, P., and Gery, I. 1997. Thymic expression of autoantigens correlates with resistance to autoimmune disease. J. Immunol. 159:3109-3112.

47. Chapoval, S.P., et al. 2002. Allergic inflammatory response to short ragweed allergenic extract in HLA-DQ transgenic mice lacking CD4 gene. J. Immunol. 168:890-899.

48. Sakaguchi, M., Sugita, S., Sagawa, K., Itoh, K., and Mochizuki, M. 1998. Cytokine production by $\mathrm{T}$ cells infiltrating in the eye of uveitis patients. Jpn. J. Ophtalmol. 42:262-268.

49. de Kozak, Y., and Verwaerde, C. 2002. Cytokine in immunotherapy of experimental uveitis. Int. Rev. Immunol. 21:231-253.

50. Abraham, R.S., and David, C.S. 2000. Identification of HLA-class-IIrestricted epitopes of autoantigens in transgenic mice. Curr. Opin. Immunol. 12:122-129.

51. Gosgrove, D., et al. 1991. Mice lacking MHC class II molecules. Cell. 66:1051-1066.

52. Lawrance, S.K., et al. 1989. Transgenic HLA-DR alpha faithfully reconstitutes IE-controlled immune functions and induces cross-tolerance to E alpha in E alpha 0 mutant mice. Cell. 58:583-594. 